\title{
Gravitational radiation from plunging orbits: Perturbative study
}

\author{
Yasushi Mino* and Jeandrew Brink ${ }^{\dagger}$ \\ California Institute of Technology, MC 130-33, Pasadena, California 91125, USA
}

(Received 16 September 2008; published 18 December 2008)

\begin{abstract}
Numerical relativity has recently yielded a plethora of results about kicks from spinning mergers which has, in turn, vastly increased our knowledge about the spin interactions of black hole systems. In this work we use black hole perturbation theory to calculate accurately the gravitational waves emanating from the end of the plunging stage of an extreme mass ratio merger in order to further understand this phenomenon. This study focuses primarily on spin induced effects with emphasis on the maximally spinning limit and the identification of possible causes of generic behavior. We find that gravitational waves emitted during the plunging phase exhibit damped oscillatory behavior, corresponding to a coherent excitation of quasinormal modes by the test particle. This feature is universal in the sense that the frequencies and damping time do not depend on the orbital parameters of the plunging particle. Furthermore, the observed frequencies are distinct from those associated with the usual free quasinormal ringing. Our calculation suggests that a maximum in radiated energy and momentum occurs at spin parameters equal to $a / M=$ 0.86 and $a / M=0.81$, respectively, for the plunge stage of a polar orbit. The dependence of linear momentum emission on the angle at which a polar orbit impacts the horizon is quantified. One of the advantages of the perturbation approach adopted here is that insight into the actual mechanism of radiation emission and its relationship to black hole ringing is obtained by carefully identifying the dominant terms in the expansions used.
\end{abstract}

DOI: 10.1103/PhysRevD.78.124015

PACS numbers: 04.30.Db, 04.25.Nx, 04.70.Bw

\section{INTRODUCTION}

As the field of numerical relativity matures, the gravitational wave community gains insight into the most dynamic regions of spacetime [1]. Possibly the most spectacular strong field event in gravity, namely, that of a binary black hole merger, has recently yielded a series of interesting results related to the spins of the holes involved in the collision. A particular highlight is the discovery of the so-called superkick configuration, where the spin interaction drastically changes the trajectory of the resultant black hole [2-4]. The body of knowledge relating to spinning compact objects has greatly increased as a result, inviting analytic explanations. Important analytic strides in this regard have been made [5-10].

The evolution of a black hole binary proceeds through three stages: the inspiral on a quasistable orbit, the plunge and merger, and the final ringdown stage. It is in the plunge and merger where the strong field fully nonlinear attraction of general relativity completely dominates the dynamics.

Of these three stages, the first and third have been carefully understood analytically. The first stage, during which the slow inspiral over a long time scale results in the emission of huge amounts of gravitational radiation that dominate the observed signal, has been extensively modeled $[11,12]$. The final stage, where the highly distorted black hole approaches a Kerr black hole via quasinormal ringing, is also carefully understood in terms of vacuum black hole perturbation theory [13-16].

\footnotetext{
*mino@tapir.caltech.edu

†jeandrew@caltech.edu
}

The dynamics of the intermediary phase of plunge and merger, where the binary transitions from a system described by several parameters related to the two black holes to a resultant system described by far fewer parameters (namely the spin, mass, and position of the resultant single black hole), has been less thoroughly explored. This transition should by its very nature display universal behavior. The interest in this phase of the binary evolution is threefold. First, the transition from inspiral to ringdown marks a turning point in the amplitude of the gravitational waveform and is essential for the production of accurate templates for observation. Second, it is thought that the plunging phase strongly influences the final kick velocity attained by the final black hole. Third, this phase gives us the opportunity to probe highly nonlinear/non-Newtonian events such as horizon formation and the merger process itself, to which currently only numerical relativity gives access.

The first issue can be addressed by attempting to extrapolate waveforms produced for the first and third stages to bridge the chasm of understanding present in the second stage. A method which extends the post-Newtonian (PN) approximation into this regime while leaning on numerical relativity results was proposed in [8,9], however it is mentioned there that the uncertainty due to the plunging stage remains considerable. The amount of linear momentum emitted during the plunge stage is expected to be significant in comparison to that emitted during inspiral, making the contribution from the final plunge stage to the final velocity of the kicks of the black hole important. Furthermore, this statement also implies that the position in the orbit at which a binary merges strongly influences 
the end result. While the kick velocities resulting from spin interactions can be modeled effectively by fitting formulas which treat the strong field interactions as an effective black box [2-4,7], a careful understanding of the interactions that produce them and the precise connection to gravitational radiation still remains somewhat elusive. A constructive formalism for treating and understanding strongly nonlinear effects in the highly dynamic regions of spacetime during the plunge and merger phases is in its early stages of development. Subtle details, exhibited in the waveform and dynamics during the merger phase, such as the antikick observed by [17] give us further clues as to dynamics that require explanation. As numerical relativity now takes the lead in ushering in a new stage in our analytic exploration of the Einstein field equations, we can begin to build our intuition about how interactions between the compact object take place and develop the tools for describing them.

In this paper, we study gravitational waves emitted from the end of the plunging phase of a small black hole falling into a spinning black hole. The purpose of the calculation is to obtain a physical understanding of the origin of the radiation emitted and to identify possible causes of universal behavior. We explore the extreme mass ratio case via black hole perturbation theory. This approach is used for two reasons. First, it makes the problem analytically tractable at minimal computational cost. Second, it gives insight into the physical mechanism by which the radiation is produced by the plunging phase of the black hole merger and directly relates that picture to the resulting gauge invariant radiation quantities computed at infinity.

The proposed model allows the effect of spin to be explored by considering the background spacetime to be a Kerr black hole with mass $M$ and spin $a$. The second smaller black hole is modeled as a point particle moving on a geodesic orbit described in Sec. II. Past studies in which black hole perturbation theory is applied to a Schwarzschild black hole have indicated that an important contribution to the energy radiated during an infall has its origin just outside the event horizon [18]. It is on this region we will focus. Since we are interested in the plunging phase of the orbit, we make use of the near-horizon approximation, which is introduced in Sec. II. Features of the final stage of the plunge that influence the emitted radiation are identified in Sec. III and Appendix B. The Teukolsky formalism is used to compute the gravitational waveform from the end of the plunging stage in Sec. III, and to compute the energy and momentum fluxes in Secs. IV and V, respectively. Numerical explorations on a Kerr background within the perturbation framework have indicated that spin enhances the radiation emission $[19,20]$. This was understood in the context of the lower damping rates of the quasinormal modes in the presence of spin [13]. In our work, the spin enhancement effect is carefully quantified analytically during the end of the plunge phase.
Our work aims at analytically quantifying features of the transition from the plunge to the ringdown phase which cannot be captured by extending purely post-Newtonian results [21]. The analysis presented here does not extend into the regime of free quasinormal ringing observed when a highly distorted black hole approaches Kerr in the absence of an external source. This is due to the fact that the perturbing second black hole is still present and therefore a source of waves.

A possible concern about modeling the end of the black hole plunge in the extreme mass ratio limit with first order perturbation theory is that the problem has been oversimplified by ignoring nonlinear perturbations and employing the point particle approximation for the smaller black hole. However, it should be observed that the dynamical time scale associated with the small black hole is comparatively short, making the equilibration process of internal perturbations of the small black hole rapid and thus justifying the point particle approximation. A huge advantage of the model is that it takes into account the fully relativistic frame-dragging effect on the orbit in the vicinity of the event horizon. A further advantage is that it allows for the exploration of the maximally spinning black hole limit. This is a case of particular theoretical interest which can only be explored by taking a limit within this analytic perturbation framework. Finally, the perturbation formalism implicitly takes into account the horizon deformation of the background spacetime, although care has to be exercised when dealing with a source that crosses the event horizon, as is shown in Appendix A. It is hoped that with the rapid advance of numerical simulations into the extreme mass ratio regime, the predictions of our model will soon be numerically tested.

The analysis presented in this paper allows us to identify the following effects. At the end of the plunging stage just before the small black hole passes through the event horizon, the radiation is dominated by a peculiar feature indicative of the ergoregion. This feature is best described as follows. In its own reference frame, the small black hole passes through the event horizon in finite time; however, to a distant observer it remains a radiating mirage stuck on the event horizon and dragged into motion at a tremendous speed, emitting gravitational waves. In practice, it is not the mirage emitting gravitational waves, but the nonlinear perturbed gravitational field that has stored up energy and angular momentum and is slowly leaking them out to the observer at infinity. The frequency and damping rate display universal behavior in that the earlier details of the orbital trajectory are irrelevant; only the position at which the small black hole crosses the horizon matters. The emitted radiation has a frequency and damping rate analogous to quasinormal ringing but at a frequency distinct from it. Pretorius [1] gave a heuristic description of the sinusoidal bobbing motion observed in the numerical simulations of two black holes in the so-called superkick 
configurations in terms of a frame-dragging phenomenon. He mentions that the position in the orbit where the merger takes place ultimately determines the magnitude and direction of the kick. While the magnitude of the kick cannot be accurately accounted for by the extreme mass ratio analysis of the plunge phase of the orbit, the dependence of the radiated linear momentum on the angular position on the horizon at which the small black hole enters can, and is calculated in Sec. V. We conclude the paper with possible observational and analytic implications in Sec. VI.

Throughout this paper, geometrized units, such as $G=$ $c=1$ are used.

\section{ORBITAL DESCRIPTION OF PLUNGING OBJECT}

In this section we describe the nature of the orbit in the final plunging phase. The rapid time scale on which the dynamics of the plunging phase occurs, implies that the orbital evolution of the small black hole is well approximated by a geodesic in the background Kerr geometry, ignoring the radiation reaction effect. Since our focus is on the late plunging phase, we then further specialize the orbit to the near-horizon region, also referred to as the nearhorizon limit.

The geodesic equations describing the trajectory of a freely falling test particle in the Kerr geometry can be expressed in Boyer-Lindquist coordinates $\{t, r, \theta, \phi\}$ using the equations [22]

$$
\left(\frac{d r}{d s}\right)^{2}=R(r), \quad\left(\frac{d \theta}{d s}\right)^{2}=\Theta(\theta)
$$

where

$$
\begin{aligned}
& R(r)=\left[E\left(r^{2}+a^{2}\right)-a L_{z}\right]^{2}-\Delta\left[\left(a E-L_{z}\right)^{2}+r^{2}+C\right], \\
& \Theta(\theta)=C-\cos ^{2} \theta\left\{a^{2}\left(1-E^{2}\right)+\left(\frac{L_{z}^{2}}{\sin ^{2} \theta}\right)\right\}
\end{aligned}
$$

and

$$
\begin{aligned}
\frac{d \phi}{d s} & =-\left(a E-\frac{L_{z}}{\sin ^{2} \theta}\right)+\frac{a}{\Delta}\left(E\left(r^{2}+a^{2}\right)-a L_{z}\right), \\
\frac{d t}{d s}= & -\left(a E-\frac{L_{z}}{\sin ^{2} \theta}\right) a \sin ^{2} \theta+\frac{r^{2}+a^{2}}{\Delta}\left(E\left(r^{2}+a^{2}\right)\right. \\
& \left.-a L_{z}\right) .
\end{aligned}
$$

In the above expressions, the constants of motion for a particular orbit are denoted $E, L_{z}$, and $C$ for energy, azimuthal angular momentum, and Carter constant respectively; furthermore, the function $\Delta$ is defined to be $\Delta=$ $r^{2}-2 M r+a^{2}$ and the affine parameter $s$ is related to the proper time $\tau$ by $s=\int d \tau / \Sigma$, with $\Sigma=r^{2}+a^{2} \cos ^{2} \theta$.

To facilitate understanding of the radiation content emanating from the orbit just before the test mass falls into the horizon, we further specialize these equations to the near- horizon limit. Denote the radial position of the event horizon by $r=r_{+}=M+\sqrt{M^{2}-a^{2}}$. The quantitative features of an in-falling orbit in the near-horizon region $\left(r \approx r_{+}\right)$can be adequately described by approximating the geodesic equations (2.1) and (2.2) by

$$
\begin{gathered}
\frac{d r}{d s}=-2 M r_{+}\left(E-\Omega_{H} L_{z}\right)+O\left(r-r_{+}\right), \\
\frac{d \theta}{d s}= \pm \sqrt{\Theta_{0}}+O\left(\theta-\theta_{0}\right), \quad \Theta_{0}=\Theta\left(\theta_{0}\right), \\
\frac{d \phi}{d s}=\frac{a}{\kappa}\left(E-\Omega_{H} L_{z}\right) \frac{r_{+}}{r-r_{+}}+O\left(\left(r-r_{+}\right)^{0}\right), \\
\frac{d t}{d s}=\frac{2 M r_{+}}{\kappa}\left(E-\Omega_{H} L_{z}\right) \frac{r_{+}}{r-r_{+}}+O\left(\left(r-r_{+}\right)^{0}\right),
\end{gathered}
$$

where $\Omega_{H}=\frac{a}{2 M r_{+}}$is the horizon's angular velocity, $\theta_{0}$ is the polar angle at which the particle falls into the horizon, and the dimensionless constant $\kappa=\sqrt{1-(a / M)^{2}}$.

The geodesic in the near-horizon limit has the following analytic solution:

$$
\begin{gathered}
r=r_{+}\left(1+e^{-\kappa\left(t-t_{0}\right) / r_{+}}\right), \quad \frac{d r}{d t}=-\kappa \frac{r-r_{+}}{r_{+}}, \\
\theta=\theta_{0} \mp \frac{\sqrt{\Theta_{0}} e^{-\kappa\left(t-t_{0}\right) / r_{+}}}{2 M\left(E-\Omega_{H} L_{z}\right)}, \\
\frac{d \theta}{d t}= \pm \frac{\kappa \sqrt{\Theta_{0}}\left(r-r_{+}\right)}{2 M r_{+}^{2}\left(E-\Omega_{H} L_{z}\right)},
\end{gathered}
$$

$$
\phi=\Omega_{H}\left(t-t_{0}\right)+\phi_{0}, \quad \frac{d \phi}{d t}=\Omega_{H},
$$

where $t_{0}$ and $\phi_{0}$ are integration constants. This approximation holds for $t-t_{0} \gg r_{+}$.

Note that the well-known gravitational time dilation effect is clearly manifest in terms of the singular behavior of Eq. (2.7). While the particle passes through the event horizon in finite proper time in its own local reference frame, it appears to a distant observer who describes the particle motion in terms of coordinate time to be slowing down infinitely as it approaches the event horizon. As a result, the motion in the radial and polar directions becomes frozen onto the event horizon at their entering positions. If the central black hole has no spin, this particle will cease to be a source of gravitational waves during the final stages of the plunge to the order of approximations made.

If the central black hole is spinning, however, Eq. (2.10) indicates that the motion in the azimuthal angle does not slow down. Instead the frame-dragging effect "pulls" the particle into motion around the black hole with an azimuthal angular velocity that approaches the horizon angular 
velocity. The particle motion around a spinning black hole in the final stage of the plunge is thus, from the perspective of a distant observer, entirely dominated by this azimuthal motion which is insensitive to the previous details of the orbital evolution and characterized only by the mass and spin of the central object. This effect is highly relativistic, it cannot be captured by an analysis based on a postNewtonian expansion, and is the cause of strong gravitational wave emission during the plunging phase of a spinning black hole. This statement will be made explicit in Sec. III.

While the constants such as $E, L_{z}$, and $C$ describing orbital motion do not enter directly into the description of the final stages of the plunging orbit itself, they do make their appearance in the dominant terms of the perturbation theory (see Appendix A) and are thus required for explicit evaluation. To this end, we have selected three plunging orbits for explicit evaluation: the co-rotating and counterrotating plunging trajectories from the innermost stable circular orbit (ISCO) in the equatorial plane (i.e. $C=0$ and $\theta_{0}=\pi / 2$ ), and the orbit plunging from the polar ISCO with the vanishing $z$ component of the angular momentum (i.e. $L_{z}=0$ ). The constants of motion characterizing these orbits can be calculated by writing down the condition that the orbit remains circular, namely, by requiring that $R\left(r, E, L_{z}, C\right)=d R\left(r, E, L_{z}, C\right) / d r=0$ at the ISCO radius $r=r_{\text {ISCO }}$. The additional condition that the orbit remains stable adds the equation $d^{2} R\left(r, E, L_{z}, C\right) / d r^{2}=0$. If one further assumes that the orbit is either polar or equatorial, namely, that $C=0$ or $L_{z}=0$, then the three equations allow the three unknowns, $E, L_{z}$, and $r=r_{\mathrm{ISCO}}$, to be expressed in terms of the black hole mass $M$ and spin $a$.

Figure 1 depicts the ISCO radius $r=r_{\text {ISCO }}$, orbital energy $E$, and azimuthal angular momentum $L_{z}$ as func- tions of the spin coefficient $q=a / M$. Because of spinorbit coupling, the orbital energy of the ISCO depends strongly on the spin coefficient when the orbit lies in the equatorial plane. When the orbit is co-rotating/counterrotating, the spin-orbit coupling works as a repulsive/attractive force that stabilizes/destabilizes the orbit with respect to the gravitational attraction of the background black hole. As a result, the radius of the co-rotating/ counter-rotating ISCO becomes smaller/larger when compared to the ISCO in the nonspinning limit. This effect is displayed in the first panel of Fig. 1. For the polar orbit which corresponds to the superkick configuration, the orbital energy of ISCO depends weakly on the spin coefficient. It turns out that only the $E$ and $L_{z}$ orbital parameters ultimately enter the waveform calculation in Sec. III. However, for completeness, the Carter constant $C$ in the case of the plunge from the polar ISCO orbit can be found in terms of energy $E$ and $r=r_{\text {ISCO }}$ to be

$$
C=E^{2} \frac{\left(a^{2}+r_{\mathrm{ISCO}}^{2}\right)^{3}\left(3 r_{\mathrm{ISCO}}^{2}-a^{2}\right)}{8\left(r_{\mathrm{ISCO}}^{2}-a^{2}\right)^{3}}
$$

\section{GRAVITATIONAL WAVES EMITTED DURING THE FINAL STAGE OF THE PLUNGE}

The gravitational waves emitted at infinity can be computed using the black hole perturbation theory expressed using the Teukolsky formalism. The radiation contained in the resulting waves is expressed in terms of the NewmanPenrose curvature scalar $\Psi_{4}$ :

$$
\Psi_{4}(r \rightarrow \infty)=\frac{1}{2}\left(\ddot{h}_{+}-i \ddot{h}_{\times}\right)
$$

where $h_{+/ \times}$represents the wave polarization and the double dot indicates two time derivatives.
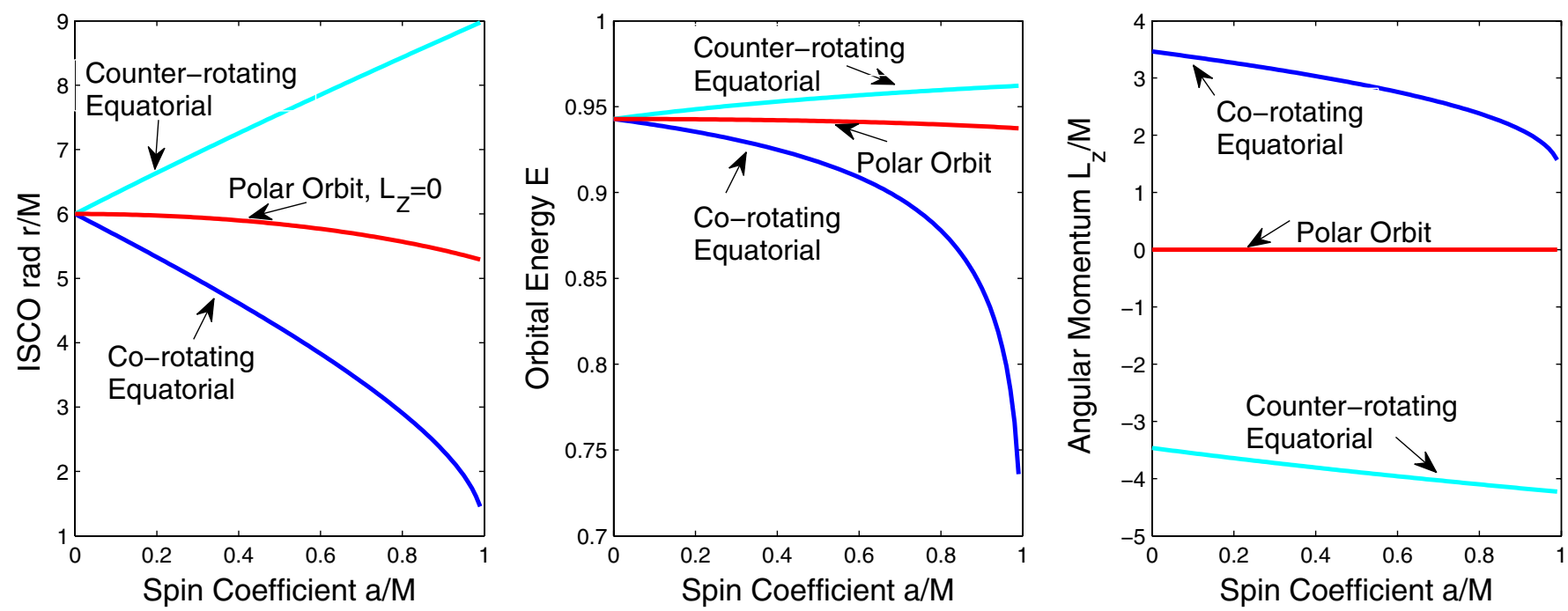

FIG. 1 (color online). ISCO radius, orbital energy $(E)$, and azimuthal angular momentum $\left(L_{z}\right)$ as a function of the spin coefficient $q=a / M$. 
Initially it may appear that, due to its bewildering complexity, the full waveform calculation of a plunging orbit within the context of black hole perturbation theory may best be attempted numerically by means of direct integration of the Teukolsky equation as was done in [18-20,23]. However, it turns out that identifying the dominant terms and spin effects in the calculation analytically is particularly insightful. Furthermore, in the rapidly spinning case, it is highly likely that numerics will not be able to capture the final stages of the plunge and that this regime is thus only accessible via analytic perturbation theory. The full Teukolsky machinery is summarized in gory detail in Appendix A. This section highlights the aspects that make the problem tractable analytically, the approximations made, and the results obtained.

The scalar $\Psi_{4}$ can be expanded in terms of its Fourierharmonic components as

$$
\Psi_{4}=\frac{1}{(r-i a \cos \theta)^{4}} \int d \omega \sum_{\ell m} e^{-i \omega t+i m \phi} R_{\ell m \omega}(r) S_{\ell m \omega}(\theta),
$$

where $R_{\ell m \omega}(r)$ and $S_{\ell m \omega}(\theta)$ are the radial and angular Teukolsky functions, respectively. The influence of the motion of the point particle as it plunges into the black hole enters the radial Teukolsky equation as a source term. The particular solution to the radial Teukolsky equation can be found by means of evaluating an integral containing a Green's function constructed from an expansion of homogeneous solutions to the Teukolsky equation. The fact that we are only interested in the solution at $r \rightarrow \infty$ simplifies the expression given in Appendix A to

$$
\begin{aligned}
R_{\ell m \omega}(r \rightarrow \infty) & \rightarrow \frac{r^{3} e^{i \omega r^{*}}}{2 i \omega B_{\ell m \omega}^{\mathrm{in}}} \int_{r_{+}}^{\infty} d r R_{\ell m \omega}^{\mathrm{in}} \Delta^{-2} T_{\ell m \omega} \\
& \rightarrow r^{3} e^{i \omega r^{*}} Z_{\ell m \omega},
\end{aligned}
$$

where $B_{\ell m \omega}^{\text {in }}$ is a mode dependent constant, $T_{\ell m \omega}$ the source term related to the stress-energy tensor describing the particle motion, and $B_{\ell m \omega}^{\mathrm{in}}$ is the homogeneous in-going Teukolsky function with asymptotic behavior

$$
R_{\ell m \omega}^{\text {in }} \rightarrow\left\{\begin{array}{ll}
B_{\ell m \omega}^{\mathrm{trans}} \Delta^{2} e^{-i k r^{*}} & \text { for } r \rightarrow r_{+} \\
B_{\ell m \omega}^{\text {ref }} r^{3} e^{i \omega r^{*}}+B_{\ell m \omega}^{\text {in }} r^{-1} e^{-i \omega r^{*}} & \text { for } r \rightarrow \infty
\end{array} .\right.
$$

In the above equation, $k=\omega-m a / 2 M r_{+}$and $r^{*}$ is the tortoise coordinate defined by $\left(d r^{*} / d r\right)=\left(r^{2}+a^{2}\right) / \Delta$.

The nature of the problem, with the point particle on the final stages of the plunging orbit, requires that this source integral need only be evaluated near the horizon to capture the essential features of the radiation emitted during this phase. Thus the asymptotic form of $R_{\ell m \omega}^{\text {in }}$ given in Eq. (3.4) can be used. It is useful to observe that in this region $\Delta \approx$ $2 \kappa M\left(r-r_{+}\right)$.

The dominant contribution to the source term results the rapid frame-dragging motion observed when the particle is frozen onto the horizon at the angle of impact $\theta_{0}$. This contribution is contained in the $T_{\overline{m m}}$ component of the stress-energy tensor projected along the tetrad legs, defined in Appendix A. The dominant scalings of the other components of the source term on the event horizon for a plunging orbit are written out in full in Appendix B.

With these approximations, the function $Z_{\ell m \omega}$ entering the asymptotic form of the radial Teukolsky function (3.3) can be evaluated to be

$$
\left.Z_{\ell m \omega}=\tilde{Z}_{\ell m \omega} \int d t e^{i \omega t-i m \phi} \frac{r-r_{+}}{r_{+}} e^{-i k r^{*}}\left\{1+O\left(\epsilon_{H}\right)\right)\right\}
$$

$$
\begin{aligned}
\tilde{Z}_{\ell m \omega}= & \mu \sqrt{\frac{2}{\pi}} \frac{1}{2 i \omega} \frac{B_{\ell m \omega}^{\mathrm{trans}}}{B_{\ell m \omega}^{\mathrm{in}}} \frac{\kappa^{3} M}{r_{+}\left(E-\Omega_{H} L_{z}\right)} \\
& \times\left[1-2 i \frac{k r_{+}}{\kappa}-2\left(\frac{k r_{+}}{\kappa}\right)^{2}\right]\left(a E-\frac{L_{z}}{\sin ^{2} \theta_{0}}\right)^{2} \\
& \times \frac{r_{+}-i a \cos \theta_{0}}{r_{+}+i a \cos \theta_{0}} \sin ^{2} \theta_{0} S_{\ell m \omega}\left(\theta_{0}\right) .
\end{aligned}
$$

where $\epsilon_{H}=\left(r-r_{+}\right) / r_{+}$.

Using the solution to the geodesic equations specialized to the region near the event horizon (2.8), (2.9), and (2.10) to evaluate $\phi$ along the orbit and $r^{*} \rightarrow\left(r_{+} / \kappa\right) \ln \left(r-r_{+}\right)$, the $t$ integral of (3.5) becomes

$$
\begin{aligned}
& \int_{T}^{\infty} d t e^{i \omega t-i m \Omega_{H} t} e^{-\left(\kappa / r_{+}\right)\left(t-t_{0}\right)} e^{i k\left(t-t_{0}\right)} \\
& =\frac{i}{2} e^{i k\left(2 T-t_{0}\right)-\left(\kappa / r_{+}\right)\left(T-t_{0}\right)} \frac{1}{\omega-m \Omega_{H}+i \frac{\kappa}{2 r_{+}}},
\end{aligned}
$$

where the lower bound of the integration, $T$, must be chosen so that $e^{-\left(\kappa / r_{+}\right)\left(T-t_{0}\right)} \ll 1$, thus, ensuring the validity of the near-horizon expansion.

From Eq. (3.7) it becomes clear that the emitted radiation is strongly peaked at the frequency $\omega=m \Omega_{H}-i \frac{\kappa}{2 r_{+}}$. Taking this into account, the asymptotic form of the curvature scalar $\Psi_{4}$ is

$$
\begin{aligned}
\Psi_{4}(r \rightarrow \infty)= & -\pi \frac{e^{\left(\kappa t_{0} / 2 r_{+}\right)}}{r} \\
& \times \sum_{\ell m}\left[e^{-i \omega\left(t-r^{*}\right)+i m \phi} S_{\ell m \omega}(\theta) \tilde{Z}_{\ell m \omega}\right],
\end{aligned}
$$

where the sum is evaluated at an angular frequency of $\omega=$ $m \Omega_{H}-i \frac{\kappa}{2 r_{+}}$. This result represents a decaying spectrum of gravitational waves with frequency around $\omega=m \Omega_{H}$ being damped in a manner that admits a quality factor of

$$
Q=\frac{|m|}{\sqrt{1-(a / M)^{2}}}\left(\frac{a}{M}\right) \text {. }
$$

The analytic results characterizing the gravitational waves from the plunging phase of the orbit yields the following observations. First, the frequency and quality 
factors of the observed radiation differ from the wellknown quasinormal ringing modes of black hole perturbation theory. The quasinormal ringing that is observed and expected to follow most binary black hole collisions can be characterized as damping modes associated with vacuum perturbations. The quasinormal ringing is often associated with the relaxation of perturbations of the stationary black hole horizon. The origin of the radiation described by Eq. (3.8) is somewhat different. It is produced by perturbations coherently induced by the particle still present on the horizon. The damping behavior is primarily due to the fact that the particle falls through the horizon and ceases to be a source of perturbation. For a quantitative comparison with the least damped ( $l=m=2)$ quasinormal mode, one can make use of a fitting formula suggested by Echeverria [14] that gives the quality factor and frequency of this mode as a function of spin to be

$$
\begin{gathered}
Q_{\mathrm{QN}}=4(1-a)^{-0.45} f_{Q}(a), \\
\omega_{\mathrm{QN}}=\left[1-0.63\left(1-a^{0.3}\right)\right] f_{f}(a),
\end{gathered}
$$

where $f_{Q}$ and $f_{a}$ are functions of order unity as the spin factor ranges from 0 to 1, i.e. $f_{Q} \in[1.05,0.95]$ and $f_{a} \in$ $[1.02,0.97]$. Note that for the spin factor $a / M<0.97$, both the frequency and quality factor of the quasinormal mode exceed those predicted by the driven motion of the plunging phase. As a result, the moment the particle ceases to be observable and thus to serve as a source driving the radiation, the quasinormal ringing effect will rapidly begin to dominate the observables. In the region of very high spin $(a / M>0.97)$ the two effects of quasinormal ringing and radiation driven by particle motion on the horizon become almost indistinguishable. In the low spin limit the difference between the frequency and quality factor of the quasinormal ringing and those associated with the radiation during plunge phase begin to differ considerably. However, it should also be noted that in this limit, our assumption that the dominant term in the stress-energy tensor expansion is the frame dragging induced rotation breaks down.

Second, while the small particle is plunging, the radiation emitted broadcasts features of the background spacetime, namely, Kerr, rather than features reminiscent of its orbital trajectory. The spectrum peak at around $\omega \sim m \Omega_{H}$ is determined entirely by the constants describing the Kerr black hole without any reference to the orbital constants of the plunge. As argued in Sec. II, the frame-dragging effect accelerates the rotational velocity of the particle around the spin axis of the black hole. The origin of the universal value of the frequency peak can thus best be explained as the frame-dragging effect compensating for the gravitational time dilation effect of the radiation.

Third, the quality factor associated with the radiation emitted during the plunging phase approaches infinity in the maximally spinning limit. The radiation emitted during the plunge in this limit may provide a sensitive means of estimating the spin of a rapidly rotating black hole. The quality factors for the radiation of gravitational waves with $(m=2)$ for a series of spin parameters are tabulated below.

\begin{tabular}{llllllll}
\hline \hline$a / M$ & 0.95 & 0.97 & 0.98 & 0.99 & 0.995 & 0.998 & 0.9999 \\
$Q$ & 6.08 & 7.98 & 9.85 & 14.04 & 19.92 & 31.58 & 141.41 \\
\hline \hline
\end{tabular}

The large quality factor obtained for the rapidly spinning limit can be used to explain the enhanced emission of gravitational energy and momentum from the plunge observed in the numerical studies made within the Teukolsky perturbation framework $[19,20]$. The feature that the quality factor approaches infinity in the maximally spinning limit may appear to endanger the assumptions made earlier regarding the insignificance of radiation reaction on the orbit. For if a mode at a finite frequency lasts an infinitely long time, it will radiate an infinite amount of energy. It should be noted that the quasinormal modes of Kerr display a similar feature. In the past, it was feared that this infinite quality factor in the case of quasinormal modes implied the instability of the Kerr black hole, however this turns out not to be the case $[13,15]$. The resolution of this conundrum with respect to the current problem is explored in Sec. IV

Fourth, the relevant expansion parameter near the horizon of the black hole is $\epsilon_{H}=\left(r-r_{+}\right) / r_{+}$. In the analysis performed in this section, only the leading order terms were retained. This approach is now justified by the following argument. Suppose one goes one step further, and computes the higher order terms of the orbit and stress energy. This will give rise to additional terms proportional to $\int^{\infty} d t e^{i \omega t-i m \Omega_{H} t} e^{-n\left(\kappa / r_{+}\right)\left(t-t_{0}\right)} e^{i k\left(t-t_{0}\right)} \propto \frac{1}{\omega-m \Omega_{H}+\operatorname{in}\left(\kappa / 2 r_{+}\right)}$ which enter the calculation for the radial Teukolsky function, Eq. (3.5), where $n=2,3, \cdots$. This suggests that the curvature perturbation in the plunging phase can more generally be written as

$$
\Psi_{4}(r \rightarrow \infty)=\frac{1}{r} \sum_{\ell m, n=1,2, \cdots}\left[e^{-i \omega\left(t-r^{*}\right)+i m \phi} S_{\ell m \omega}(\theta) \tilde{X}_{\ell m \omega}\right],
$$

where the frequency $\omega$ entering the expression is different for every mode and given by $\omega=m \Omega_{H}-$ in $\frac{\kappa}{2 r_{+}}$. Note that in the above equation, the damping factor has been enhanced by a factor of $n$ and so the dynamics described thus far are truly the dominant behavior, since all higher order terms are more strongly damped.

\section{ENERGY FLUX}

The picture presented thus far is that of a small body falling into the central black hole on a dynamical time scale with respect to proper time, which for sufficiently extreme mass ratios, is assumed to be well short of the radiation reaction time scale. This justifies the assumption that gravitational radiation backaction on the orbit is neg- 
ligible and that the particle plunges on a geodesic trajectory.

In Eq. (3.9) of Sec. II, it was shown that for rapidly spinning black holes, the quality factor becomes very large in the maximally spinning limit. This fact could possibly indicate that the gravitational wave emission at the horizon angular frequency for a maximally rotating black hole may be considerable and last for a long period of time. If this were the case, it would nullify the assumptions previously made regarding the geodesic trajectory of the plunging black hole.

In this section, we calculate the gravitational energy flux during the final stage of the plunge and estimate the maximum amount of energy the particle can emit during the plunging phase. In doing so, we check the validity of the assumptions made and explore the possibility of direct detection of this stage of the orbit.

The gravitational energy flux at infinity can be expressed as

$$
\begin{aligned}
\frac{d E}{d t} & =\lim _{r \rightarrow \infty}\left[\frac{r^{2}}{16 \pi} \int d \Omega\left|\int d t \Psi_{4}\right|^{2}\right] \\
& \rightarrow \frac{\pi^{2}}{8} e^{-\left(\kappa / r_{+}\right)\left(t-t_{0}-r^{*}\right)} \sum_{\ell m} \frac{\left|\tilde{Z}_{\ell m \bar{\omega}_{m}}\right|^{2}}{\left|\bar{\omega}_{m}\right|^{2}},
\end{aligned}
$$

where $\bar{\omega}_{m}=m \Omega_{H}-i \frac{\kappa}{2 r_{+}}$is used. The total energy radiated from $t-r^{*}=T$ to $t-r^{*}=\infty$ is given by

$$
\begin{aligned}
\Delta E= & \frac{\pi^{2}}{8} e^{-\left(\kappa / r_{+}\right)\left(T-t_{0}\right)} \frac{r_{+}}{\kappa} \sum_{\ell m} \frac{\left|\tilde{Z}_{\ell m \bar{\omega}_{m}}\right|^{2}}{\left|\bar{\omega}_{m}\right|^{2}} \\
= & \frac{\pi}{16} \mu^{2} \frac{\kappa^{5} M^{2}}{r_{+}} e^{-\left(\kappa / r_{+}\right)\left(T-t_{0}\right)} \frac{\left(a E-\frac{L_{z}}{\sin ^{2} \theta_{0}}\right)^{4}}{\left(E-\Omega_{H} L_{z}\right)^{2}} \\
& \times \sin ^{4} \theta_{0} \sum_{\ell m} \frac{\left|S_{\ell m \bar{\omega}_{m}}\left(\theta_{0}\right)\right|^{2}}{\left|\bar{\omega}_{m}\right|^{4}}\left|\frac{B_{\ell m \bar{\omega}_{m}}^{\mathrm{trans}}}{B_{\ell m \bar{\omega}_{m}}^{\text {in }}}\right|^{2} .
\end{aligned}
$$

Equation (4.2) can be evaluated by using the analytic expressions for the homogeneous Teukolsky functions in the low-frequency limit given in Appendix C. It can be observed that the relative contribution of higher $\ell$ modes converge very rapidly; in fact, they fall off superexponentially, allowing us to consider only the $\ell=2$ modes. So doing we have

$$
\begin{aligned}
\Delta E \approx & \frac{\pi}{1024} \mu^{2} \frac{\kappa^{11} M^{2}}{r_{+}^{7}} e^{-\left(\kappa / r_{+}\right)\left(T-t_{0}\right)} \frac{\left(a E-\frac{L_{z}}{\sin ^{2} \theta_{0}}\right)^{4}}{\left(E-\Omega_{H} L_{z}\right)^{2}} \sin ^{4} \theta_{0} \\
& \times\left\{\frac{8}{45}+\left(\frac{32}{15}-\frac{8}{5} \sin ^{2}\left(\theta_{0}\right)\right)\left(\frac{a}{\kappa M}\right)^{2}\right. \\
& +\left(\frac{128}{15}-8 \sin ^{2}\left(\theta_{0}\right)+\frac{4}{5} \sin ^{4}\left(\theta_{0}\right)\right)\left(\frac{a}{\kappa M}\right)^{4} \\
& \left.+\left(\frac{512}{45}-\frac{56}{5} \sin ^{2}\left(\theta_{0}\right)+\frac{4}{3} \sin ^{4}\left(\theta_{0}\right)\right)\left(\frac{a}{\kappa M}\right)^{6}\right\}
\end{aligned}
$$

In the maximally spinning limit, the scaling behavior of the total radiated energy with respect to the quality factor can be expressed as

$$
\Delta E \propto \kappa^{5} \propto Q^{-5} .
$$

This suggests that even though gravitational waves could be emitted for an extremely long time at a finite frequency in the maximally spinning limit, the wave amplitude of this radiation is suppressed and the resultant total energy radiated small. This shows conclusively that if the mass ratio is sufficiently extreme, the backaction due to the emission of gravitational waves is negligible. The approximation of the spacetime geometry by the linear metric perturbation theory remains valid, as does the assumption that the in-falling particle moves on a geodesic orbit. This result further implies that it will be difficult to observe highly relativistic dynamics of the particle orbiting just outside the horizon in the maximally spinning case because of the strong suppression of the wave amplitude.

The observed suppression of the wave amplitude in the maximally spinning case can be explained using the radial Teukolsky equation (A5). Near the horizon the homogeneous radial Teukolsky equation (A5) becomes

$$
\left(r-r_{+}\right) \frac{d}{d r}\left(\frac{1}{r-r_{+}} \frac{d R_{\ell m \omega}}{d r}\right)+\frac{\left(k r_{+}\right)^{2}+2 i \kappa\left(k r_{+}\right)}{\kappa^{2}\left(r-r_{+}\right)^{2}} R_{\ell m \omega}=0 .
$$

The pole in the potential has a coefficient of $\kappa^{-2}$. As a result, in the maximally rotating limit $(\kappa \rightarrow 0)$ it becomes increasingly difficult for radiation originating from a source near the horizon to propagate outward and reach infinity. The potential barrier in the Teukolsky equation thus produces a suppression effect that competes with the high quality factor of emitted radiation described in Sec. II and ultimately dominates as the black hole becomes maximally spinning. The competition between these two effects result in a spin coefficient less than unity at which the maximum amount of energy is radiated during a plunge. This feature is present in all orbits explored.

Figure 2 displays the total energy radiated during the plunging stage of orbits originating from ISCO. The factor $e^{-\left(\kappa / r_{+}\right)\left(T-t_{0}\right)}$ present in Eq. (4.3) is weakly dependent on the orbital trajectory and set to unity for simplicity. Figure 2 plot (a) shows the energy radiated if the original orbit is equatorial. The nonzero $z$ component of the angular momentum $\left(L_{z}\right)$ results in finite energy being radiated in the limit of zero spin, i.e. $a / M=0$. As the background spin increases from zero, the radiated energy of the corotating plunging orbit decreases. This effect is due to an effective decrease in the angular momentum resulting from the frame dragging of the background black hole-the factor of $\left(a E-L_{z}\right)^{4}$ in Eq. (4.3) makes this apparent. In the counter-rotating case, the same effect results in a net increase in energy radiated as spin increases from zero. As the spin further increases, the competition between the 

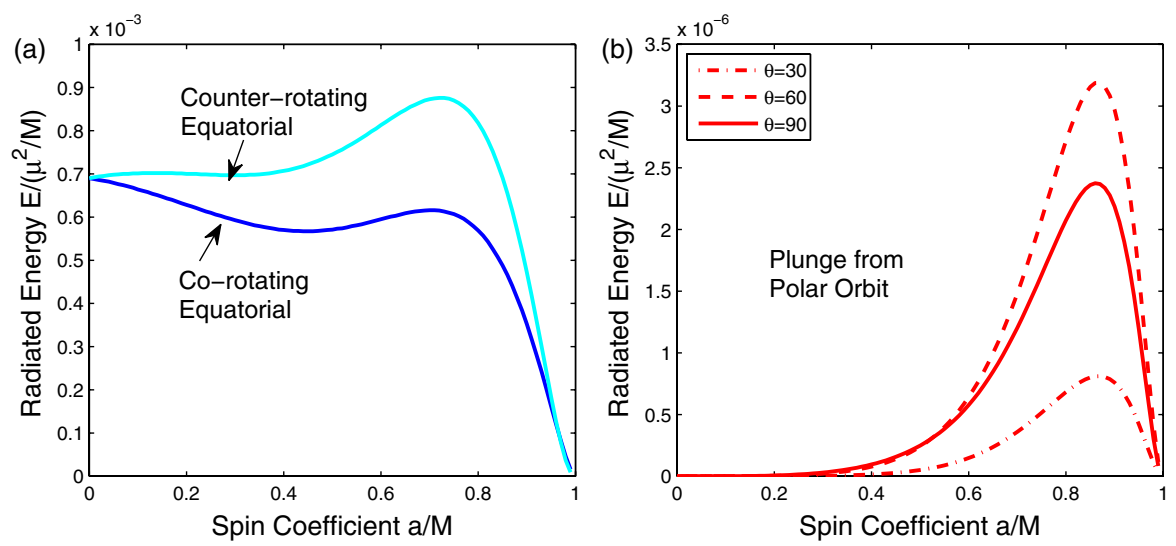

FIG. 2 (color online). Total energy radiated during the plunging phase of orbit from ISCO as a function of spin parameter. Plot (a) displays the energy radiated when plunging from an equatorial ISCO $(C=0)$ in the co-rotating and counter-rotating cases. Plot (b) shows the radiated energy if the particle originates from a polar orbit for various horizon impact angles, $\theta=\frac{\pi}{6}, \frac{\pi}{3}, \frac{\pi}{2}$.

increasing quality factor associated with the radiation and the suppression effect due to the effective potential barrier, described in the previous paragraph, sets in. A maximum in radiated energy is achieved at $a / M=0.7$ and $a / M=0.72$ for the co-rotating and counter-rotating cases, respectively.

Figure 2 plot (b) shows the energy radiated from the end of the plunging phase when the particle plunges from a polar ISCO orbit. The azimuthal angular momentum $\left(L_{z}\right)$ vanishes for this case and the resultant energy radiated approaches zero in the limit of zero spin, $a / M=0$. As we increase the spin of the background black hole, an effective angular momentum represented by the factor $\left(a E-L_{z}\right)^{4}$ in Eq. (4.3) comes into play and the radiated energy increases. For particles plunging from a polar orbit, the radiation suppression effect noted in the equatorial case is also observed for high spin factors and a maximum in radiated energy is reached. This peak occurs at a spin factor of $a / M=0.87$ when the impact angle with the horizon is $\theta_{0}=\pi / 6$ or $\theta_{0}=\pi / 3$ and at $a / M=0.86$ when the impact angle is $\theta_{0}=\pi / 2$. The best fit to the slopes of the three energy profiles in Fig. 2 plot (b) for the range of spin factor between $0.7<a / M<0.8$ is found to be $\Delta E=$ $3.22 \times 10^{-6}(a / M)-18.94 \times 10^{-6}$ for $\theta_{0}=\pi / 6, \Delta E=$ $1.24 \times 10^{-5}(a / M)-7.20 \times 10^{-6}$ for $\theta_{0}=\pi / 3$, and $\Delta E=0.87 \times 10^{-5}(a / M)-5.89 \times 10^{-6}$ for $\theta_{0}=\pi / 3$.

In the case of a particle plunging from a polar orbit, the radiated energy is dependent on the angle made with the spin axis when the particle impacts the horizon. The angular dependence of the radiated energy is displayed in Fig. 3 for various values of spin parameter.

Observe that the maximum in radiated energy does not occur when the particle falls in on the equatorial plane, $\theta_{0}=\pi / 2$, but that it occurs instead for impact angles of $\theta_{0}=1.07$ and $\theta_{0}=2.06$. One would naively expect to find that the radiated energy is largest in the configuration where the particle has maximum orbital velocity $v$ when it impacts the horizon, namely, on the equatorial plane at $\theta_{0}=\pi / 2$. An initial scaling argument would be that the energy radiated is roughly proportional to the square of the metric perturbation which in turn is proportional to the square of the stress-energy tensor of the source term $T \propto$ $v^{2} \propto(\omega r)^{2} \sin ^{2} \theta_{0}$. This effect is indeed displayed by the appearance of the factor of $\sin ^{4} \theta_{0}$ in Eq. (4.3). The energy emission is, however, complicated by the angular pattern resulting from the spin-2 nature of gravitational waves, which is described by spheroidal harmonics that enter Eq. (4.3). The origin of the double maxima in the emitted energy can be explained by focusing on the $(\ell m)$ sum of Eq. (4.2). Making use of Eq. (C5), to evaluate the ratio $B^{\text {trans }} / B^{\text {in }}$, the sum can be expressed as

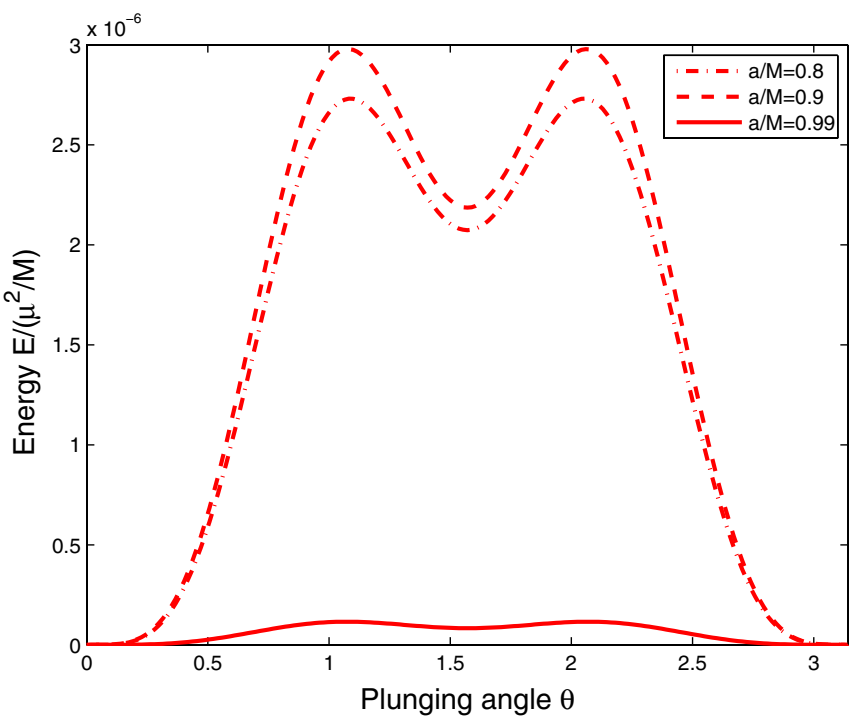

FIG. 3 (color online). Total energy radiated during the plunging phase of a particle originating on a polar ISCO as a function of impact angle with the horizon. Radiation for background Kerr black holes with spin parameter $(a / M)=0.8,0.9,0.99$ are plotted. 


$$
\sum_{\ell m}\left|\bar{\omega}_{m}\right|^{6}\left|S_{\ell m \bar{\omega}_{m}}\right|^{2},
$$

with $\operatorname{Re}\left[\bar{\omega}_{m}\right]=m \Omega_{H}$. The dominant contribution to the sum in Eq. (4.2) and thus the radiated energy is made by modes with the largest $|m|$ value for a given $\ell$. If we consider the contribution from $(\ell=2)$ modes using the low-frequency result in Appendix $\mathrm{C}$, the $\theta_{0}$ dependence becomes

$$
\sin ^{4}\left(\theta_{0}\right)\left\{\left(1-\cos \left(\theta_{0}\right)\right)^{4}+\left(1+\cos \left(\theta_{0}\right)\right)^{4}\right\},
$$

which has two maxima at $\theta_{0}=1.06$ and $\theta_{0}=2.08$ and a local minima at $\theta_{0}=\pi / 2$, and thus accurately explains the $\theta_{0}$ dependence observed in Fig. 3.

\section{LINEAR MOMENTUM FLUX}

During the evolution of a typical binary system, the longest period of time is spent in the inspiral phase. It is during this phase that most of the gravitational energy is emitted. By comparison to the inspiral phase the energy flux, computed in Sec. IV, emitted during the plunging phase is insignificant. The situation is very different when one considers the linear momentum flux. The net linear momentum emission during the inspiral phase is small, because the binary orbit gradually progresses through a series of quasistable circular orbits resulting in nearly isotropic momentum emission. As a result, the momentum emitted during the plunging stage is important and may be comparable to that emitted during the inspiraling phase [10]. In this section we quantify the linear momentum flux carried away by gravitational waves during the final plunge phase of a spinning black hole.

The emitted linear momentum flux is given by

$$
\begin{aligned}
\frac{d P_{i}}{d t}= & \lim _{r \rightarrow \infty}\left[\frac{r^{2}}{16 \pi} \int d \Omega n_{i}\left|\int_{-\infty}^{t} d t \Psi_{4}\right|^{2}\right] \\
\rightarrow & \frac{\pi^{2}}{8} e^{-\left(\kappa / r_{+}\right)\left(t-t_{0}-r^{*}\right)} \sum_{\ell m, \ell^{\prime} m^{\prime}} \frac{e^{-i\left(m-m^{\prime}\right) \Omega_{H}\left(t-r^{*}\right)}}{\bar{\omega}_{m}\left(\bar{\omega}_{m^{\prime}}\right)^{*}} \frac{1}{2 \pi} \\
& \times \int d \Omega n_{i} e^{i\left(m-m^{\prime}\right) \phi} S_{\ell m \bar{\omega}_{m}}\left(S_{\ell^{\prime} m^{\prime} \bar{\omega}_{m^{\prime}}}\right)^{*} \tilde{Z}_{\ell m \bar{\omega}_{m}}\left(\tilde{Z}_{\ell^{\prime} m^{\prime} \bar{\omega}_{m^{\prime}}}\right)^{*},
\end{aligned}
$$

where $n_{i}=(\sin \theta \cos \phi, \sin \theta \sin \phi, \cos \theta)$.

The $z$ component of the momentum flux, $d P_{z} / d t$, vanishes near the horizon. This is yet another manifestation of the phenomenon discussed in Sec. II, where the particle is frozen onto the horizon due to the time dilation effect and its motion is dominated by the velocity component in the $\phi$ direction. In the near-horizon expansion of the source term given in Appendix B, at the leading order only, the particle motion in the $x$ and $y$ directions are taken into account and the net emission of $z$-linear momentum vanishes as a result.

The calculated total linear momentum carried away by gravitational waves during the period $T<t-r^{*}<\infty$ is

$$
\begin{aligned}
\Delta\left(P_{x}+i P_{y}\right)= & \frac{\pi}{16} \mu^{2} \frac{\kappa^{5} M^{2}}{r_{+}} \frac{e^{-\left(\kappa / r_{+}\right)\left(T-t_{0}\right)-i \Omega_{H} T}}{1-i \frac{a}{2 \kappa M}} \frac{\left(a E-\frac{L_{z}}{\sin ^{2} \theta_{0}}\right)^{4}}{\left(E-\Omega_{H} L_{z}\right)^{2}} \sin ^{4} \theta_{0} \sum_{\ell \ell^{\prime} m} \frac{1}{\bar{\omega}_{m}^{2}\left(\left(\bar{\omega}_{m^{\prime}}\right)^{*}\right)^{2}} S_{\ell m \bar{\omega}_{m}}\left(\theta_{0}\right)\left(S_{\ell^{\prime} m^{\prime} \bar{\omega}_{m^{\prime}}}\left(\theta_{0}\right)\right)^{*} \\
& \times\left(\frac{B_{\ell m \bar{\omega}_{m}}^{\mathrm{trans}}}{B_{\ell m \bar{\omega}_{m}}^{\text {in }}}\right)\left(\frac{B_{\ell^{\prime} m^{\prime} \bar{\omega}_{m^{\prime}}}^{\mathrm{trans}}}{B_{\ell^{\prime} m^{\prime} \bar{\omega}_{m^{\prime}}}^{\text {in }}}\right)^{*} \int_{0}^{\pi} d \theta \sin ^{2}(\theta) S_{\ell m \bar{\omega}_{m}}(\theta)\left(S_{\ell^{\prime} m^{\prime} \bar{\omega}_{m^{\prime}}}(\theta)\right)^{*},
\end{aligned}
$$

where we set $m^{\prime}=m+1$. To facilitate explicit evaluation, the analytic expressions for the Teukolsky functions in the low frequency given in Appendix $\mathrm{C}$ are used. The resulting expression is

$$
\begin{aligned}
\Delta\left(P_{x}+i P_{y}\right) \approx & \frac{\pi}{1024} \mu^{2} \frac{\kappa^{11} M^{2}}{r_{+}^{7}} \frac{e^{-\left(\kappa / r_{+}\right)\left(T-t_{0}\right)-i \Omega_{H} T+i(a / 2 \kappa M) \ln \kappa}}{1-i \frac{a}{2 \kappa M}} \frac{\left(a E-\frac{L_{z}}{\sin ^{2} \theta_{0}}\right)^{4}}{\left(E-\Omega_{H} L_{z}\right)^{2}} \sin ^{5} \theta_{0}\left\{\frac{16}{135}-i \frac{16}{45}\left(\frac{a}{\kappa M}\right)\right. \\
& +\left(\frac{16}{45}-\frac{8}{15} \sin ^{2}\left(\theta_{0}\right)\right)\left(\frac{a}{\kappa M}\right)^{2}+i\left(-\frac{176}{135}+\frac{16}{15} \sin ^{2}\left(\theta_{0}\right)\right)\left(\frac{a}{\kappa M}\right)^{3}+\left(\frac{32}{45}-\frac{8}{15} \sin ^{2}\left(\theta_{0}\right)\right)\left(\frac{a}{\kappa M}\right)^{4} \\
& \left.+i\left(-\frac{64}{45}+\frac{16}{15} \sin ^{2}\left(\theta_{0}\right)\right)\left(\frac{a}{\kappa M}\right)^{5}+\left(\frac{128}{135}-\frac{32}{45} \sin ^{2}\left(\theta_{0}\right)\right)\left(\frac{a}{\kappa M}\right)^{6}\right\} .
\end{aligned}
$$

The momentum radiated during the final plunging phase of an orbit originating at ISCO on the equatorial plane is plotted in Fig. 4(a). Analogous to the radiated energy of the same orbit, Fig. 2(a), the linear momentum plotted here displays a finite value in the limit of a nonspinning Kerr background hole due to the orbital angular momentum of the plunging particle. The momentum radiation further displays features reminiscent of the radiated energy, in that the radiated momentum decreases for the co-rotating plunge and displays an increase at first followed by a decrease for the counter-rotating plunge as the background spin increases from zero. A feature distinct from those displayed by the radiated energy in Fig. 2(a) is that no local maxima of the radiated momentum is apparent. This 
is due to the factor $1 /(1-i(a / 2 \kappa M))$ which comes from the time integration of the momentum flux. This can be better understood by observing that in Eq. (5.1), the momentum flux displays damped oscillatory behavior and for large spin, the oscillation frequency becomes high. When taking the integral to obtain the total radiated momentum, the positive and negative parts of the integrand cancel, resulting in a small value for the integral. This stands in contrast to the radiated energy where the integrand is a positive decaying function without any oscillatory behavior.

The oscillatory behavior of the momentum flux made explicit in Eq. (5.1) indicates that the linear momentum flux is emitted in various directions in the $x / y$ plane. This feature is to be expected if the particle is in effect pulled into orbit around the black hole as it plunges and so the radiated momentum has an oscillatory nature that will reflect the horizon velocity of the final black hole. A similar process was alluded to in Fig. 14 in the second reference of [9] to explain the antikick phenomena observed in [17].

The momentum radiated during the final stages of plunge from a polar ISCO is shown in Fig. 4(b). Analogous to the radiated energy plotted in Fig. 2(b), the radiated momentum increases from zero as the background spin increases. The radiated momentum reaches a maximum at $a / M=0.82$, when the in-falling angle is $\theta_{0}=$ $\pi / 6$ or $\theta_{0}=\pi / 3$ and at $a / M=0.81$ when the in-falling angle is $\theta_{0}=\pi / 2$. As the spin factor ranges over $0.55<$ $a / M<0.75$, the radiated momentum can be fitted by $\Delta P=1.23 \times 10^{-7}(a / M)-5.65 \times 10^{-8}$ for $\theta_{0}=\pi / 6$ $\Delta P=0.95 \times 10^{-6}(a / M)-4.12 \times 10^{-7}$ for $\theta_{0}=\pi / 3$, and $\Delta P=0.94 \times 10^{-6}(a / M)-3.62 \times 10^{-7}$ for $\theta_{0}=$ $\pi / 3$.

The dependence of the radiated momentum on the angular position at which the particle impacts the horizon

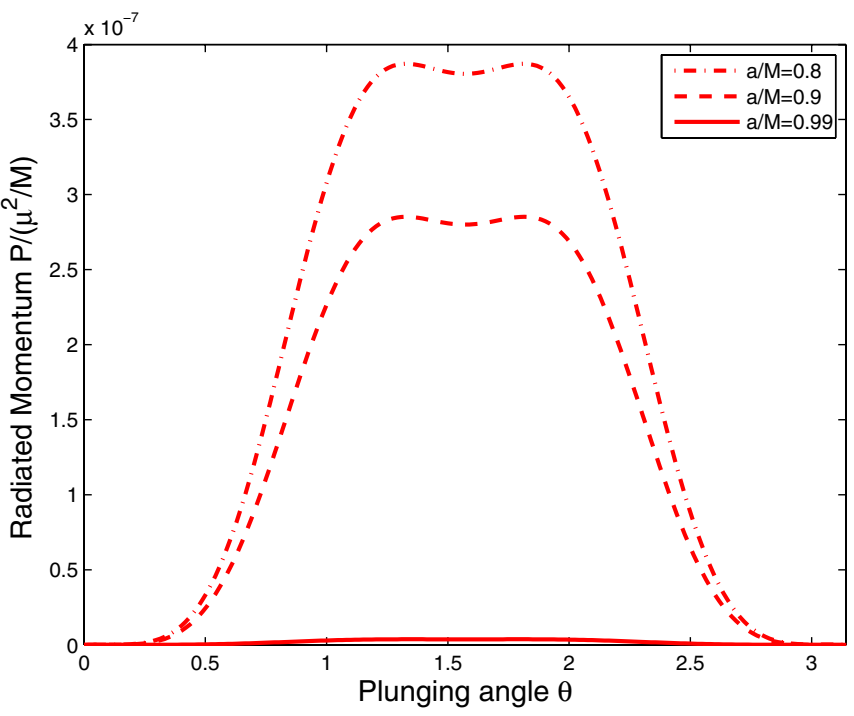

FIG. 5 (color online). Total momentum radiated during the plunging phase of a particle originating on a polar ISCO as a function of impact angle with the horizon. Radiation for background Kerr black holes with spin parameter $(a / M)=0.8,0.9$, 0.99 are plotted.

during a plunge from a polar orbit is show in Fig. 5. The angular profiles of the radiated momentum (Fig. 5) and those of the radiated energy (Fig. 3) differ slightly. The features of the local minimum at $\theta_{0}=\pi / 2$ and the two maxima off the equatorial plane are robust. For the case of radiated momenta, the maxima occur at $\theta_{0}=1.33$ and $\theta_{0}=1.82$. Following an argument similar to that employed in the case of the radiated energy, the observed behavior can be explained by analyzing the $\theta_{0}$ dependence of the $\left(\ell \ell^{\prime} m\right)$ sum in the first line of Eq. (5.2). The dominant functional behavior is found to be

$$
\sin ^{5}\left(\theta_{0}\right)\left\{\left(1-\cos \left(\theta_{0}\right)\right)^{3}+\left(1+\cos \left(\theta_{0}\right)\right)^{3}\right\}
$$
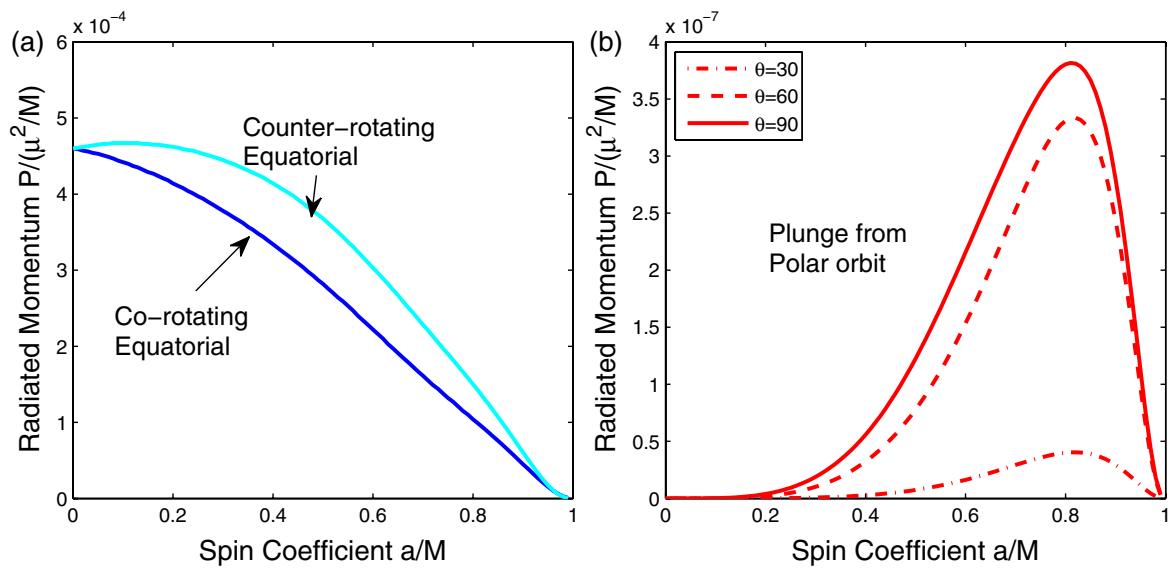

FIG. 4 (color online). Total momentum radiated during the final plunging phase of orbit at ISCO as a function of spin parameter. Plot (a) displays the momentum radiated when plunging from an equatorial ISCO $(C=0)$ in the co-rotating and counter-rotating cases. Plot (b) shows the radiated momentum if the particle originates from a polar orbit $\left(L_{z}=0\right)$ for various horizon impact angles, $\theta=\frac{\pi}{6}$, $\frac{\pi}{3}, \frac{\pi}{2}$. 
which admits maxima at $\theta_{0}=1.35$ and $\theta_{0}=1.79$ and a local minima at $\theta_{0}=\pi / 2$.

\section{CONCLUSION}

This paper explores the dynamics of the final stages of the plunge of a small black hole into a Kerr black hole and characterizes the resulting gravitational radiation, using perturbation theory. The purpose of this exploration is to gain analytic understanding of the features which dominate the dynamics of this transition phase of the merger process before the onset of quasinormal ringing and to identify any universal features. Our analysis strives to highlight the effects of the spin of the central black hole on the resulting gravitational waveform and momentum flux while building an intuitive picture of the processes responsible for their origin. It should be noted that all graphs plotted in this paper and comments made are relevant only to radiation emanating from the so-called near-horizon region during the plunging phase.

In Sec. III, it was found that the waveform resulting from the plunging phase can be expressed as

$$
h(t)=\sum_{n, m=1,2 \ldots} h_{(m, n)} e^{-i \omega_{(m, n)} t},
$$

where the frequencies entering the expansion take on the universal form $\omega_{(m, n)}=m \Omega_{H} t-$ in $\kappa /\left(2 r_{+}\right)$. Therefore, the time dependence of the emitted radiation is entirely determined by the characteristics of the massive central black hole. This waveform describes the transition period between radiation dominated by the inspiral and radiation dominated by the free quasinormal ringing. The inspiral stage broadcasts the characteristic frequencies of the orbiting particle and is strongly affected by the constants of motion describing the orbit. The quasinormal ringing phase is characterized by the frequencies and damping rates associated with free vacuum perturbations of an excited Kerr black hole as it settles down to equilibrium. The frequencies that enter expression (6.1) can be ascribed to neither of the two preceding scenarios. They describe a state of forced oscillation distinct in frequency from free quasinormal ringing but are at the same time devoid of any characteristic identifying the source of the perturbation. In fact, the source can be best described as a reluctant mirage on the horizon-a mirage dragged into motion and then retained for distant observation by an all-dominating frame-dragging effect. The frequency associated with $\omega_{(m, n)}$ originates from the azimuthal motion of the orbit on the horizon, which is entirely determined by parameters of the background black hole. The method by which this mirage is stripped of its identity while it in essence passes through the event horizon and is assimilated into the large black hole is described in mathematical detail in Sec. II.

The wave amplitude $h_{(m, n)}$ still retains characteristics of the source and depends on the orbital constants. The damping rate associated with $\omega_{(m, n)}$ indicates that the radiation corresponds to a decaying part of the waveform. A feature that this frequency shares with the quasinormal mode frequencies is the existence of a high quality factor in the maximally spinning limit $a / M \rightarrow 1$, for example, $Q=$ 14.04 for $a / M=0.99$ and $Q=141.41$ for $a / M=0.99$. A more detailed comparison between the least damped quasinormal mode frequency and $\omega_{(2,1)}$ is given toward the end of Sec. III.

The gravitational flux of energy and linear momentum originating from the plunging phase is computed in Secs. IV and V, respectively. Orbits plunging from ISCO in the equatorial plane (i.e. $C=0$ ) and from a polar ISCO (i.e. $L_{z}=0$ ) have been considered. In general, the following characteristics can be identified. The rapid increase in quality factor is counteracted by a term in the amplitude $h_{(m, n)}$ that rapidly approaches zero as spin approaches maximality. This ensures that all forms of radiation approach zero in the limit of maximal spin and that the total emitted energy and momentum during this phase remain finite. This effect further ensures that a maximum in emitted energy as a function of spin is attained in all cases considered. For the plunge from an equatorial orbit the maximum in radiated energy occurs around $a / M=0.7$ and for the plunge from polar orbit the maximum occurs for a spin parameter around $a / M=0.86$.

The plunge from a polar ISCO orbit is of particular interest because it can be used as a toy problem to illuminate some of the features observed in numerical simulations of black hole mergers in superkick configurations. In particular, the dependence of the emitted linear momentum on the angle $\theta_{0}$ made with the rotation axis when the particle enters the horizon is computed in this paper. Contrary to expectation, it was found in Sec. V that the produced profile does not have a simple sinusoidal dependence on angle, but rather that it is complicated by the spin2 nature of gravitational radiation, as displayed in Fig. 5.

The radiated linear momentum originating from a polar plunge reaches a maximum with respect to the spin parameter at around $a / M=0.86$. While the origin of this peak is well understood within the context of our model, the question remains as to, whether the peak is an artifact of linear perturbation theory and our underlying assumptions or whether one will find its counterpart in numerical simulations. We also find that the radiated energy and linear momentum can be fitted by linear functions of $a / M$ in the domain $0.7<a / M<0.8$ and $0.55<a / M<$ 0.75 , respectively.

A final observation regarding the computed momentum flux of Sec. V Eq. (5.1) is the existence of oscillatory behavior with a frequency we now consider characteristic of a plunging event involving spin. The introduced phase implies that the linear momentum flux is emitted in various directions in the $x / y$ plane, consistent with our picture of a miraged particle stuck on the event horizon radiating momentum in a manner that reflects the velocity of the final 
black hole while it slowly recedes from view. This could provide a heuristic explanation of the origin of the antikick phenomenon observed in [17] and is suggestive that the antikick is indicative of the final merger event.

\section{ACKNOWLEDGMENTS}

We would like to thank Professor Kip Thorne, Professor Yanbei Chen, and Dr. Emanuele Berti for fruitful discussions. Y. M. is supported by NSF Grant No. PHY-0601459, No. PHY-0653653, NASA Grant No. NNX07AH06G, No. NNG04GK98G, and the Brinson Foundation. J. B. gratefully acknowledges support from the Fairchild Foundation.

\section{APPENDIX A: TEUKOLSKY FORMALISM}

This appendix summarizes the Teukolsky formalism used in the derivation of the Weyl scalar $\Psi_{4}$ which contains the radiation content of the perturbed spacetime with a prescribed source term.

The scalar $\Psi_{4}$ can be decomposed into its Fourierharmonic components as follows:

$$
\Psi_{4}=\frac{1}{(r-i a \cos \theta)^{4}} \sum_{\ell m} \int d \omega e^{-i \omega t+i m \phi} R_{\ell m \omega}(r) S_{\ell m \omega}(\theta),
$$

where $S_{\ell m \omega}(\theta)$ and $R_{\ell m \omega}(r)$ represent the angular and radial Teukolsky functions, respectively. The angular function obeys the angular Teukolsky equation for $s=-2$, explicitly,

$$
\frac{1}{\sin \theta} \frac{d}{d \theta}\left(\sin \theta \frac{d S_{\ell m \omega}}{d \theta}\right)-U S_{\ell m \omega}=0
$$

where the potential $U$ is given by

$$
\begin{aligned}
U= & a^{2} \omega^{2} \sin ^{2} \theta+\frac{(m-2 \cos \theta)^{2}}{\sin ^{2} \theta}-4 a \omega \cos \theta+2 \\
& -2 m a \omega-\lambda,
\end{aligned}
$$

and $\lambda$ denotes the eigenvalue of $S_{\ell m \omega}$. The angular Teukolsky function is normalized so that

$$
\int_{0}^{\pi} \sin \theta d \theta\left|S_{\ell m \omega}\right|^{2}=1 .
$$

The radial Teukolsky function in turn satisfies

$$
\Delta^{2} \frac{d}{d r}\left(\frac{1}{\Delta} \frac{d R_{\ell m \omega}}{d r}\right)-V R_{\ell m \omega}=T_{\ell m \omega}
$$

where the potential $V$ is defined to be

$$
V=-\frac{K^{2}+4 i(r-M) K}{\Delta}+8 i \omega r+\lambda
$$

and the function $K=\left(r^{2}+a^{2}\right) \omega-m a$. The source term $T_{\ell m \omega}$ contains the effect of the stress-energy tensor perturbing the background spacetime. The relationship be- tween the source term and the stress-energy tensor is given by

$$
T_{\ell m \omega}=4 \int d \Omega d t \rho^{-5} \bar{\rho}^{-1}\left(T_{1}+T_{2}\right) e^{-i m \phi+i \omega t} \frac{S_{\ell m \omega}}{\sqrt{2 \pi}},
$$

$$
\begin{aligned}
T_{1}= & -\frac{1}{2} \rho^{8} \bar{\rho} \mathcal{L}_{-1}\left[\rho^{-4} \mathcal{L}_{0}\left(\rho^{-2} \bar{\rho}^{-1} T_{n n}\right)\right] \\
& -\frac{1}{2 \sqrt{2}} \rho^{8} \bar{\rho} \Delta^{2} \mathcal{L}_{-1}\left[\rho^{-4} \bar{\rho}^{2} \mathcal{D}_{+}\left(\rho^{-2} \bar{\rho}^{-2} \Delta^{-1} T_{\bar{m} n}\right)\right],
\end{aligned}
$$

$$
\begin{aligned}
T_{2}= & -\frac{1}{4} \rho^{8} \bar{\rho} \Delta^{2} \mathcal{D}_{+}\left[\rho^{-4} \mathcal{D}_{+}\left(\rho^{-2} \bar{\rho} T_{\bar{m} \bar{m}}\right)\right] \\
& -\frac{1}{2 \sqrt{2}} \rho^{8} \bar{\rho} \Delta^{2} \mathcal{D}_{+}\left[\rho^{-4} \bar{\rho}^{2} \Delta^{-1} \mathcal{L}_{-1}\left(\rho^{-2} \bar{\rho}^{-2} T_{\bar{m} n}\right)\right],
\end{aligned}
$$

where we use $\rho=(r-i a \cos \theta)^{-1}, \bar{\rho}=(r+i a \cos \theta)^{-1}$, and the operators $\mathcal{D}_{+}=\partial_{r}+i K / \Delta$, and $\mathcal{L}_{s}=$ $\partial_{\theta}+\frac{m}{\sin \theta}-a \omega \sin \theta+s \cot \theta$. The terms $T_{n n}, T_{\bar{m} n}$, and $T_{\bar{m} \bar{m}}$ denote the tetrad components of the stress-energy tensor. In this paper, a point particle was used as the source of the metric perturbation. The stress-energy tensor associated with a point particle can be expressed as

$$
\begin{aligned}
T^{\mu \nu}= & \frac{\mu}{\sum \sin \theta}\left(\frac{d t}{d \tau}\right)^{-1} \frac{d z^{\mu}}{d \tau} \frac{d z^{\nu}}{d \tau} \delta(r-r(t)) \\
& \times \delta(\theta-\theta(t)) \delta(\phi-\phi(t)),
\end{aligned}
$$

where $\mu$ is the mass of the particle. Using the geodesic equations (2.1) and (2.2), the source term can be simplified to

$$
\begin{aligned}
T_{l m \omega}= & \mu \int d t e^{i \omega t-i m \phi} \Delta^{2}\left[\partial_{r}^{2}\left\{A_{\bar{m} \bar{m} 2} \delta(r-r(t))\right\}\right. \\
& +\partial_{r}\left\{\left(A_{\bar{m} n 1}+A_{\bar{m} \bar{m} 1}\right) \delta(r-r(t))\right\} \\
& \left.+\left(A_{n n 0}+A_{\bar{m} n 0}+A_{\bar{m} \bar{m} 0}\right) \delta(r-r(t))\right],
\end{aligned}
$$

where

$$
\begin{aligned}
A_{n n 0}= & \frac{-2}{\sqrt{2 \pi} \Delta^{2}} B_{n n} \rho^{-2} \bar{\rho}^{-1} \mathcal{L}_{1}^{\dagger}\left\{\rho^{-4} \mathcal{L}_{2}^{\dagger}\left(\rho^{3} S\right)\right\}, \\
A_{\bar{m} n 0}= & \frac{2}{\sqrt{\pi} \Delta} B_{\bar{m} n} \rho^{-3}\left[\left(\mathcal{L}_{2}^{\dagger} S\right)\left(\frac{i K}{\Delta}+\rho+\bar{\rho}\right)\right. \\
& \left.-a \sin \theta S \frac{K}{\Delta}(\bar{\rho}-\rho)\right]
\end{aligned}
$$

$$
A_{\bar{m} \bar{m} 0}=-\frac{1}{\sqrt{2 \pi}} \rho^{-3} \bar{\rho} B_{\bar{m} \bar{m}} S\left[-i\left(\frac{K}{\Delta}\right)_{, r}-\frac{K^{2}}{\Delta^{2}}+2 i \rho \frac{K}{\Delta}\right]
$$

$$
A_{\bar{m} n 1}=\frac{2}{\sqrt{\pi} \Delta} \rho^{-3} B_{\bar{m} n}\left[\mathcal{L}_{2}^{\dagger} S+i a \sin \theta S(\bar{\rho}-\rho)\right],
$$




$$
\begin{gathered}
A_{\bar{m} \bar{m} 1}=-\frac{2}{\sqrt{2 \pi}} \rho^{-3} \bar{\rho} B_{\bar{m} \bar{m}} S\left(i \frac{K}{\Delta}+\rho\right), \\
A_{\bar{m} \bar{m} 2}=-\frac{1}{\sqrt{2 \pi}} \rho^{-3} \bar{\rho} B_{\bar{m} \bar{m}} S,
\end{gathered}
$$

and

$$
\begin{gathered}
B_{n n}=\frac{1}{4 \Sigma^{3} \dot{t}}\left[E\left(r^{2}+a^{2}\right)-a L_{z}+\Sigma \frac{d r}{d \tau}\right]^{2}, \\
B_{\bar{m} n}=-\frac{\rho}{2 \sqrt{2} \Sigma^{2} \dot{t}}\left[E\left(r^{2}+a^{2}\right)-a L_{z}+\Sigma \frac{d r}{d \tau}\right] \\
\times\left[i \sin \theta\left(a E-\frac{L_{z}}{\sin ^{2} \theta}\right)\right], \\
B_{\bar{m} \bar{m}}=\frac{\rho^{2}}{2 \Sigma \dot{t}}\left[i \sin \theta\left(a E-\frac{L_{z}}{\sin ^{2} \theta}\right)\right]^{2} .
\end{gathered}
$$

Here the operator $\mathcal{L}_{s}^{\dagger}$ is defined by $\mathcal{L}_{s}^{\dagger}=\partial_{\theta}-\frac{m}{\sin \theta}+$ $a \omega \sin \theta+s \cot \theta$, indices have been dropped from the angular Teukolsky function, i.e. $S=S_{\ell m \omega}$ and $\dot{t}=$ $d t / d \tau=(1 / \Sigma)(d t / d s)$.

For a given source the particular solution of the radial Teukolsky equation can be found by means of a Green's function method. To this end, define two homogeneous solutions to the radial Teukolsky equation which satisfy the boundary conditions

$$
\begin{gathered}
R_{\ell m \omega}^{\mathrm{in}} \rightarrow\left\{\begin{array}{ll}
B_{\ell m \omega}^{\mathrm{trans}} \Delta^{2} e^{-i k r^{*}} & \text { for } r \rightarrow r_{+} \\
B_{\ell m \omega}^{\mathrm{ref}} r^{3} e^{i \omega r^{*}}+B_{\ell m \omega}^{\mathrm{in}} r^{-1} e^{-i \omega r^{*}} & \text { for } r \rightarrow \infty
\end{array},\right. \\
R_{\ell m \omega}^{\mathrm{up}} \rightarrow\left\{\begin{array}{ll}
C_{\ell m \omega}^{\mathrm{up}} e^{i k r^{*}}+C_{\ell m \omega}^{\mathrm{ref}} \Delta^{2} e^{-i k r^{*}} & \text { for } r \rightarrow r_{+} \\
C_{\ell m \omega}^{\mathrm{trans}} r^{3} e^{i \omega r^{*}} & \text { for } r \rightarrow \infty
\end{array},\right.
\end{gathered}
$$

in these expressions $k=\omega-m a / 2 M r_{+}$and $r^{*}$ is the tortoise coordinate defined by $\left(d r^{*} / d r\right)=\left(r^{2}+a^{2}\right) / \Delta$. In terms of homogeneous solutions the particular solution of the radial Teukolsky function can be expressed as

$$
\begin{aligned}
R_{\ell m \omega}= & \frac{1}{W_{\ell m \omega}} R_{\ell m \omega}^{\mathrm{up}} \int_{r_{+}}^{r} d r R_{\ell m \omega}^{\mathrm{in}} \Delta^{-2} T_{\ell m \omega} \\
& +\frac{1}{W_{\ell m \omega}} R_{\ell m \omega}^{\mathrm{in}} \int_{r}^{\infty} d r R_{\ell m \omega}^{\mathrm{up}} \Delta^{-2} T_{\ell m \omega},
\end{aligned}
$$

where the Wronskian $W_{\ell m \omega}$ is given by $W_{\ell m \omega}=$ $2 i \omega B_{\ell m \omega}^{\text {in }} C_{\ell m \omega}^{\text {trans }}$. Of particular interest for the purposes of gravitational wave extraction is the nature of the solution at infinity,

$$
\begin{aligned}
R_{\ell m \omega}(r \rightarrow \infty) & \rightarrow \frac{r^{3} e^{i \omega r^{*}}}{2 i \omega B_{\ell m \omega}^{\mathrm{in}}} \int_{r_{+}}^{\infty} d r R_{\ell m \omega}^{\mathrm{in}} \Delta^{-2} T_{\ell m \omega} \\
& =r^{3} e^{i \omega r^{*}} Z_{\ell m \omega} .
\end{aligned}
$$

In the event that the source term is a point particle moving along a geodesic, such as the scenario considered in this paper, the function $Z_{\ell m \omega}$ can be expressed as

$$
\begin{aligned}
Z_{\ell m \omega}= & \frac{\mu}{2 i \omega B_{\ell m \omega}^{\text {in }}} \int d t e^{i \omega t-i m \phi(t)} \\
& \times\left[X_{\ell m \omega}+X_{\ell m \omega \infty}-X_{\ell m \omega+}\right],
\end{aligned}
$$

where

$$
\begin{aligned}
X_{\ell m \omega}= & {\left[R_{\ell m \omega}^{\mathrm{in}}\left\{A_{n n 0}+A_{\bar{m} n 0}+A_{\bar{m} \bar{m} 0}\right\}\right.} \\
& \left.-\frac{d R_{\ell m \omega}^{\mathrm{in}}}{d r}\left\{A_{\bar{m} n 1}+A_{\bar{m} \bar{m} 1}\right\}+\frac{d^{2} R_{\ell m \omega}^{\mathrm{in}}}{d r^{2}} A_{\bar{m} \bar{m} 2}\right]_{r=r(t)}, \\
X_{\ell m \omega \infty}= & \lim _{r(t) \rightarrow \infty} e^{i \omega t-i m \phi(t)}\left[R_{\ell m \omega}^{\mathrm{in}}\left\{A_{\bar{m} n 1}+A_{\bar{m} \bar{m} 1}\right\}\right. \\
& \left.-\left\{i\left(\omega \frac{d t}{d r}-m \frac{d \phi}{d r}\right) R_{\ell m \omega}^{\mathrm{in}}+2 \frac{d R_{\ell m \omega}^{i n}}{d r}\right\} A_{\bar{m} \bar{m} 2}\right] \frac{d t}{d r},
\end{aligned}
$$

$$
\begin{aligned}
X_{\ell m \omega+}= & \lim _{r(t) \rightarrow r_{+}} e^{i \omega t-i m \phi(t)}\left[R_{\ell m \omega}^{\mathrm{in}}\left\{A_{\bar{m} n 1}+A_{\bar{m} \bar{m} 1}\right\}\right. \\
& \left.-\left\{i\left(\omega \frac{d t}{d r}-m \frac{d \phi}{d r}\right) R_{\ell m \omega}^{\mathrm{in}}+2 \frac{d R_{\ell m \omega}^{\mathrm{in}}}{d r}\right\} A_{\bar{m} \bar{m} 2}\right] \frac{d t}{d r},
\end{aligned}
$$

the terms (A27) and (A28) appear as the boundary terms when Eq. (A24) is integrated by parts. If the source particle orbit is bound within the radial domain, namely, somewhere between infinity and the horizon, these terms vanish.

For the source under consideration in the present paper, care has to be exercised since the orbit crosses the horizon and the term (A28) may contribute to the integral. It is, however, found that this term too vanishes, which is reasonable since no waves propagate from the horizon out to infinity.

\section{APPENDIX B: LEADING ORDER BEHAVIOR OF SOURCE TERMS}

The source terms generated by a particle plunging on a geodesic orbit near the horizon of a black hole display the following leading order behavior when expanded with respect to distance from the horizon

$$
\begin{aligned}
B_{n n} \rightarrow & O\left(\left(r-r_{+}\right)^{3}\right), \quad B_{\bar{m} n} \rightarrow O\left(\left(r-r_{+}\right)^{2}\right), \\
B_{\bar{m} \bar{m}} \rightarrow & -\frac{a^{2} \kappa E_{\mathrm{ISCO}}}{4 M r_{+}} \frac{\sin ^{2} \theta_{0}}{\left(r_{+}-i a \cos \theta_{0}\right)^{2}} \frac{r-r_{+}}{r_{+}} \\
+ & O\left(\left(r-r_{+}\right)^{2}\right),
\end{aligned}
$$




$$
\begin{gathered}
A_{n n 0} \rightarrow O\left(r-r_{+}\right), \quad A_{\bar{m} n 0} \rightarrow O\left(\left(r-r_{+}\right)^{0}\right), \\
A_{\bar{m} \bar{m} 0 \rightarrow}-\frac{\kappa a^{2} E_{\mathrm{ISCO}}}{4 \sqrt{2 \pi} M r_{+}^{2}}\left\{i \frac{k r_{+}}{\kappa}+\left(\frac{k r_{+}}{\kappa}\right)^{2}\right\} \\
\quad \times \frac{r_{+}-i a \cos \theta_{0}}{r_{+}+i a \cos \theta_{0}} \sin ^{2} \theta_{0} S \frac{1}{r-r_{+}} \\
+O\left((r-r+)^{0}\right), \\
A_{\bar{m} \bar{m} 1}^{\rightarrow} \quad i \frac{\kappa a^{2} E_{\mathrm{ISCO}}}{2 \sqrt{2 \pi} M r_{+}^{2}} \frac{k r_{+}}{\kappa} \frac{r_{+}-i a \cos \theta_{0}}{r_{+}+i a \cos \theta_{0}} \sin ^{2} \theta_{0} S \\
+O(r-r+), \\
+O\left(r-r_{+}\right), \\
A_{\bar{m} \bar{m} 2}=\frac{\kappa a^{2} E_{\mathrm{ISCO}}}{4 \sqrt{2 \pi} M r_{+}^{2}} \frac{r_{+}-i a \cos \theta_{0}}{r_{+}+i a \cos \theta_{0}} \sin ^{2} \theta_{0} S\left(r-r_{+}\right) \\
+O\left((r-r+)^{2}\right) .
\end{gathered}
$$

Note that the dominant contribution of the particle's geodesic motion enters the source terms by means of $B_{\bar{m}} \bar{m}$. This term dominates due to the rapid azimuthal velocity induced by the frame-dragging effect.

\section{APPENDIX C: TEUKOLSKY FUNCTIONS IN THE LOW-FREQUENCY LIMIT}

This appendix summarizes the analytic considerations required to compute the functional form of homogeneous Teukolsky functions in the low-frequency limit.

\section{Angular Teukolsky functions}

The solutions to the angular Teukolsky equation (A2) are also known as spin-weighted angular spheroidal functions. The analytic structure of these functions was studied in [24]. By expanding the spin-weighted spheroidal functions in terms of Jacobi polynomials the angular Teukolsky equation can be reduced to three-term recurrence relations that are given by Eqs. (21-24) of [24]. The solutions of the recurrence relations were shown, in Eq. (25) of [24], to converge very rapidly with respect to an expansion in frequency, i.e. with respect to expansion parameter $a \omega$. As a result, in the low-frequency limit, Jacobi polynomials accurately capture the analytic behavior of the spinweighted spheroidal harmonics.

To leading order in the low-frequency limit, the angular Teukolsky functions can thus be well approximated by spin-weighted spherical harmonics. It is this approximation that is adopted in this paper to aid computational simplicity. In particular we have

$$
S_{\ell m \omega}(\theta) \rightarrow{ }_{-2} Y_{l m}(\theta, 0), \quad \lambda \rightarrow(\ell-1)(\ell+2),
$$

where $\ell \geq|m|$ and $\ell=2,3, \cdots$. The low $\ell$, spin-weighted spherical harmonics used in the computation are now given

$$
\begin{aligned}
& -2 Y_{2 \pm 2}(\theta, 0)=\frac{1}{8} \sqrt{\frac{5}{\pi}}(1 \pm \cos \theta)^{2}, \\
& -2 Y_{2 \pm 1}(\theta, 0)=\frac{1}{4} \sqrt{\frac{5}{\pi}} \sin \theta(1 \pm \cos \theta),
\end{aligned}
$$

$$
-2 Y_{20}(\theta, 0)=\frac{1}{4} \sqrt{\frac{15}{2 \pi}} \sin ^{2} \theta .
$$

\section{Radial Teukolsky functions}

The properties of the solutions to the radial Teukolsky equation used to aid calculation are briefly summarized in this section. The analysis of the analytic structure of the radial Teukolsky equation was first performed by Leaver [25] and further developed by Mano et al. [26]; it is this reference to which the reader should refer for a treatment more detailed than the synopsis given here.

The approach adopted in the analysis is to expand the homogeneous solutions in terms of two different series expansions, each strongly convergent at a particular boundary condition. Near the horizon hypergeometric functions were used (refer to Sec. 2 of Ref. [26]) to capture the behavior of ingoing and outgoing waves and implement the boundary condition on the horizon. An expansion in Coulomb functions was then used to give an asymptotic expression at infinity, and to fix the boundary condition of no ingoing waves (refer to Sec. 3 of Ref. [26]). These two expansions were then matched in the interior to obtain a solution valid over the whole domain obeying the appropriate boundary conditions on both boundaries (refer to Sec. 4 of Ref. [26]).

Following an approach similar to that employed in the case of the angular Teukolsky function, the radial Teukolsky equation can be reduced to three-term recurrence relations valid for both expansions. Once again the solution to the three-term recurrence relations shows very rapid convergence with respect to a low-frequency expansion. In particular the expansion parameter $\epsilon=2 \omega M$ is introduced.

In the calculation performed in this paper, the ratio of the incoming wave amplitude $B^{\text {in }}$ to the amplitude of the transmitted wave $B^{\text {trans }}$ as defined in Eq. (A21) is required. Using [26] the ratio of these wave amplitudes can be obtained in terms of a power series expansion in $\epsilon$. The rapid convergence of the expansions employed in [26] with respect to $\epsilon$ allows us to consider only the leading order term in the expansion to make a quantitative estimate of the required ratio. The wave amplitude ratio so calculated is 


$$
\begin{aligned}
\frac{B^{\text {trans }}}{B^{\text {in }}}= & i^{1-\ell} 2^{\ell+3} \omega^{5} e^{-i(m / 2)(q / \kappa) \ln \kappa}(\kappa \epsilon)^{\ell-2} \\
& \times \frac{(\ell+2) !(\ell-2) !}{(2 \ell) !(2 \ell+1) !} \frac{\Gamma\left(\ell+1+i m \frac{q}{\kappa}\right)}{\Gamma\left(3+i m \frac{q}{\kappa}\right)}(1+O(\epsilon)),
\end{aligned}
$$

where $q=a / M$ and $\kappa=\sqrt{1-q^{2}}$. The factorials in the denominator of Eq. (C5) cause the computed ratio to decrease very rapidly as $\ell$ increases. As a result only the lower $\ell$ contributions need be considered. Specifically, the lowest two terms contributing to the expression are computed to be

$$
\left[\frac{B^{\text {trans }}}{B^{\text {in }}}\right]_{\ell=2}=-i \frac{4}{15} \omega^{5} e^{-i(m / 2)(q / \kappa) \ln \kappa}(1+O(\epsilon)),
$$

$$
\begin{aligned}
{\left[\frac{B^{\text {trans }}}{B^{\text {in }}}\right]_{\ell=3}=} & -\frac{2}{945} \omega^{5}(\kappa \epsilon) e^{-i(m / 2)(q / \kappa) \ln \kappa}\left(3+i m \frac{q}{\kappa}\right) \\
& \times(1+O(\epsilon)) .
\end{aligned}
$$

[1] Frans Pretorius, Physics of Relativistic Objects in Compact Binaries: From Birth to Coalescence, edited by M. Colpi, P. Casella, V. Gorini, U. Moschella, and A. Possenti, Astrophysics and Space Science Library Vol. 359 (unpublished).

[2] M. Campanelli, C. O. Lousto, Y. Zlochower, and D. Merritt, Astrophys. J. 659, L5 (2007); J. A. Gonzalez, M. D. Hanna, U. Sperhake, B. Brugmann, and S. Husa, Phys. Rev. Lett. 98, 231101 (2007); M. Campanelli, C. O. Lousto, Y. Zlochower, and D. Merritt, Phys. Rev. Lett. 98, 231102 (2007); B. Brugmann, J. Gonzalez, M. Hannam, S. Husa, and U. Sperhake, Phys. Rev. D 77, 124047 (2008).

[3] J. G. Baker, W. D. Boggs, J. Centrella, B. J. Kelly, S. T. McWilliams, M. C. Miller, and J. R. van Meter, Astrophys. J. 682, L29 (2008).

[4] S. Dain, C. O. Lousto, and Y. Zlochower, Phys. Rev. D 78, 024039 (2008).

[5] J. D. Bekenstein, Astrophys. J. Lett. 183, 657 (1973).

[6] M. J. Fitchett, Mon. Not. R. Astron. Soc. 203, 1049 (1983); A. G. Wiseman, Phys. Rev. D 46, 1517 (1992).

[7] L. E. Kidder, Phys. Rev. D 52, 821 (1995); S. H. Miller and R. A. Matzner, arXiv:0807.3028 [Gen. Rel. Grav. (to be published)]

[8] L. Blanchet, M. S. S. Qusailah, and C. M. Will, Astrophys. J. 635, 508 (2005); T. Damour and A. Gopakumar, Phys. Rev. D 73, 124006 (2006).

[9] J.D. Schnittman and A. Buonanno, Astrophys. J. Lett. 662, L63 (2007); J. D. Schnittman, A. Bounanno, J. R. van Meter, J. G. Baker, W. D. Boggs, J. Centrella, B. J. Kelly, and S. T. McWilliams, Phys. Rev. D 77, 044031 (2008).

[10] M. J. Fitchett and S. D. Detweiler, Mon. Not. R. Astron. Soc. 211, 933 (1984); M. Favata, S. A. Hughes, and D. E. Holz, Astrophys. J. Lett. 607, L5 (2004).

[11] L. Blanchet, Living Rev. Relativity 9, 4 (2006), http:// www.livingreviews.org/lrr-2006-4, and references therein.

[12] M. Sasaki and H. Tagoshi, Living Rev. Relativity 6, 6
(2003), http://www.livingreviews.org/lrr-2003-6; Y. Mino, M. Sasaki, M. Shibata, H. Tagoshi, and T. Tanaka Prog, Theor. Phys. Suppl. 128, 1 (1997), and references therein.

[13] S. Chandrasekhar and S. L. Detweiler, Proc. R. Soc. A 344, 441 (1975); S. L. Detweiler, Proc. R. Soc. A 352, 381 (1977); S. L. Detweiler, Astrophys. J. 239, 292 (1980); E. W. Leaver, Proc. R. Soc. A 402, 285 (1985).

[14] F. Echeverria, Phys. Rev. D 40, 3194 (1989).

[15] V. Ferrari and B. Mashhoon, Phys. Rev. Lett. 52, 1361 (1984).

[16] H. P. Nollert, Phys. Rev. D 47, 5253 (1993).

[17] M. Koppitz, D. Pollney, C. Reisswig, L. Rezzolla, J. Thornburg, P. Diener, and E. Schnetter, Phys. Rev. Lett. 99, 041102 (2007); D. Pollney, C. Reisswig, L. Rezzolla, B. Szilagyi, M. Ansorg, B. Deris, P. Diener, E. N. Dorband, M. Koppitz, A. Nagar, and E. Schnetter, Phys. Rev. D 76, 124002 (2007).

[18] M. Davies, R. Ruffini, W. H. Press, and R. H. Press, Phys. Rev. Lett. 27, 1466 (1971); M. Davies, R. Ruffini, and J. Tiomno, Phys. Rev. D 5, 2932 (1972).

[19] T. Nakamura and M. Sasaki, Phys. Lett. A 89, 185 (1982); T. Nakamura and M. Haugan, Astrophys. J. 269, 292 (1983); M. Sasaki and T. Nakamura, Prog. Theor. Phys. 67, 1788 (1982).

[20] Y. Kojima and T. Nakamura, Phys. Lett. A 96, 335 (1983); Prog. Theor. Phys. 71, 79 (1984).

[21] M. Sasaki, Prog. Theor. Phys. 69, 815 (1983).

[22] B. Carter, Phys. Rev. 174, 1559 (1968).

[23] K. Oohara and T. Nakamura, Prog. Theor. Phys. 70, 757 (1983).

[24] E. D. Fackerell and R. G. Crossman, J. Math. Phys. (N.Y.) 18, 1849 (1977).

[25] E. W. Leaver, J. Math. Phys. (N.Y.) 27, 1238 (1986).

[26] S. Mano, M. Suzuki, and E. Takasugi, Prog. Theor. Phys. 95, 1079 (1996). 